\title{
A FORMAÇÃO DE PESOUISADORES NA TEMÁTICA DA SAÚDE DO TRABALHADOR DE ENFERMAGEM
}

Fábio José Silva ${ }^{1}$, Ana Paula Pelegrini Ratier ${ }^{2}$, Vanda Elisa Andres Felli ${ }^{3}$, Renata Santos Tito ${ }^{4}$, Patricia Campos Pavan Baptista ${ }^{4}$

Objetivo: verificar a formação máxima dos pesquisadores promovida por um grupo de pesquisa. Metodologia: estudo documental, descritivo de abordagem quantitativa, realizado com participantes do grupo de estudos sobre a saúde dos trabalhadores de enfermagem e saúde (GESTES). Foram coletados dados do período de 1997 a 2014 , por meio de busca online de currículo lattes dos membros e submetidos à análise estatística descritiva. Resultados: participaram do grupo 50 mulheres e seis homens. Aproximadamente 40\% dos integrantes concluíram a graduação, 36\% o mestrado, 14\% o doutorado e 4\% o pósdoutorado. Identificou-se a alta inserção dos doutores e dos mestres em instituições de ensino superior, tanto públicas como privadas. Ainda, verificou-se que a formação de mestres e doutores é dependente dos recursos humanos disponibilizados pelo programa de pós-graduação, como orientadores. Conclusão: o grupo cumpriu a sua finalidade, tanto em relação à formação numérica de pesquisadores, quanto à promoção da sua inserção em universidades.

Descritores: Capacitação de Recursos Humanos em Saúde, Saúde do Trabalhador, Pesquisa em Enfermagem, Grupos de Pesquisa.

\section{THE TRAINING OF RESEARCHERS IN THE ISSUE OF HEALTH OF THE WORKING NURSE}

Objective: to evaluate the highest training level of researchers of a research group. Methodology: documentary and descriptive study, with participants from the study group on the health of nursing and health workers (GESTES). From 1997 to 2014 , data were collected through on-line search of members' lattes curriculum and submitted to descriptive statistical analysis. Results: Fifty women and six men participated in GESTES in this period. Approximately $40 \%$ of the members concluded undergraduate level, followed by masters (36\%) and doctors (14\%) degrees. Both doctors and masters are dedicated to higher education in public or private universities. The qualification of masters and doctors is dependent on the human resources available by the pos-graduate program and mentors. Conclusion: GESTES fulfilled its purpose, in regards to the training of a considerable number of researches and in the promotion of their inclusion in universities.

Descriptors: Health Human Resource Training, Occupational Health, Nursing Research, Research Group.

\section{LA FORMACIÓN DE INVESTIGADORES EN LA TEMÁTICA DE LA SALUD DEL TRABAJADOR DE ENFERMERÍA}

Objetivo: evaluar las especificaciones de la planta física de los lugares de almacenamiento de residuos químicos peligrosos generados en hospital. Metodología: Investigación descriptiva realizada en el Hospital Universitario de la Universidad de São Paulo. La recolección de datos se realizó mediante Hoja de Información de Gestión de Residuos Químicos Peligrosos. Resultados: Los datos mostraron que 11 especificaciones, 7 (63.63\%) del almacenamiento interno no estaba en conformidad y 6 (54,54\%) cumplian los requisitos de almacenamiento externo. Los resultados también mostraron los aspectos que deben ser revisados por el Hospital, como la adecuación de la planta física, los lugares de almacenamiento de residuos químicos peligrosos, en espera del transporte externo. También fueron ofrecidas directrices como normas para la construcción de depósitos, apoyo al Plan de Gestión de Residuos Químicos Peligrosos. Conclusión: Con el Plan se hizo posible la prevención de accidentes y enfermedades relacionadas al trabajo y a la promoción de la salud humana y ambiental, como factores de sostenibilidad en el sector salud hospitalario.

Descriptores: Capacitación de Recursos Humanos en Salud, Salud Laboral, Investigación en Enfermería, Grupos de Investigación.

1Enfermeiro. Doutor em Ciências. Servidor Hospital Universitário da Universidade de São Paulo-USP. ${ }^{2}$ Terapeuta Ocupacional. Mestre em Ciências. Servidora do Hospital Universitário da USP.

${ }^{3}$ Enfermeira. Livre Docente. Professora da Escola da Escola de Enfermagem da USP. E-mail: vandaeli@usp.br ${ }^{4}$ Enfermeira. Mestre em Ciências. Servidora do Instituto do Coração. 


\section{INTRODUÇÃO}

Desde o início da pós-graduação em enfermagem, no Brasil, na década de 1970(1), a evolução do conhecimento técnico-científico na área da enfermagem tem demonstrado a execução de práticas assistenciais especializadas e embasadas em evidências científicas, oriundas de avanços no campo da pesquisa ${ }^{(2)}$. O desenvolvimento da enfermagem no campo da pesquisa científica tem sido respaldado pelos resultados de projetos realizados por grupos de pesquisa (GP), vinculados aos diversos programas de pós-graduação em enfermagem ${ }^{(3)}$.

Os GP, compostos por equipes de pesquisadores titulados ou em formação, contribuem para o fortalecimento da enfermagem como ciência e profissão. É a produção científica, gerada pelas pesquisas, que permite $o$ aprofundamento e a consolidação do conhecimento, direcionando os profissionais para práticas mais qualificadas, repercutindo em maior visibilidade à profissão(4).

Nessa realidade, percebemos o crescente número de pesquisas relacionadas à temática saúde do trabalhador de enfermagem, desde a primeira tese defendida no pais ${ }^{(5)} \mathrm{e}$, mais recentemente, na sua interface com a segurança do paciente. Isso porque considera-se que o bem-estar biopsíquico do trabalhador impacta fortemente na qualidade da assistência prestada e, portanto, na segurança do paciente( ${ }^{(6)}$.

Nessa concepção, constatamos o surgimento e o crescimento de grupos de pesquisadores que se propõem a aprofundar estudos sobre a saúde do trabalhador de enfermagem. Como resultados dos estudos, verificase também 0 incremento dos estudos que propõem a implementação de estratégias protetoras da saúde dos trabalhadores de enfermagem, visando proporcionarthes melhor qualidade de vida, ascensão na carreira e, consequentemente, melhor produtividade ${ }^{(3)}$.

Aliados à geração de conhecimento em áreas especificas, os grupos de pesquisa constituem a porta de entrada para a formação de pesquisadores, em todos os niveis de formação, ou seja, doutores, mestres, alunos de especialização e de iniciação científica. Os GP têm participação fundamental na formação de recursos humanos com importante contribuição, tanto no ensino, como na pesquisa. O crescimento dos grupos de pesquisa no Brasil demonstra contributos de inovação à ciência e tecnologia, à medida que também dão suporte à evolução dos programas de pós-graduação. Na enfermagem, os GPs demandam maior inclusão dos alunos de graduação, reforçando as estratégias de formação, tanto para a atuação na área assistencial, como no ensino e pesquisa em enfermagem ${ }^{(4,7-9)}$.

Portanto, no intuito de aprofundarmos o conhecimento e dar visibilidade aos produtos relativos à formação nessa temática pelo grupo intitulado "Grupo de Estudos sobre a Saúde dos Trabalhadores de Enfermagem (GESTE)", este estudo foi realizado com o objetivo verificar a formação máxima dos pesquisadores promovida pelo grupo de pesquisa.

\section{METODOLOGIA}

O estudo, do tipo documental, descritivo e de abordagem quantitativa, buscou verificar a formação dos pesquisadores do GESTE. Esse grupo é vinculado ao Programa de Pós Graduação em Gerenciamento em Enfermagem, do Departamento de Orientação Profissional, da Escola de Enfermagem da Universidade de São Paulo. O grupo está inscrito no Conselho Nacional de Desenvolvimento Científico e Tecnológico (CNPq).

Foram identificados 56 membros do Grupo, formados no período de 1997 a 2014, por meio das 261 publicações científicas. Essas publicações consistiram em livros, capítulos de livros, teses, dissertações e resumos em anais de congresso, trabalhos de conclusão de curso (TCC), monografias de iniciação científica (IC), monografias de especialização (ME), dissertações de mestrado (M), teses de doutorado (D) e de pós-doutorado (PD).

A coleta de dados foi realizada por meio de busca online, durante o ano de 2015, nos currículos Lattes dos membros que participaram do GESTE, desde seu surgimento, em 1997, até 2014. Foi considerada a maior titulação do pesquisador e a produção científica sobre a temática saúde do trabalhador, durante sua participação no grupo.

Os dados foram inseridos em planilha Excel $\odot$, sistematizados e analisados estatisticamente utilizando-se frequência relativa e absoluta. Após a análise, os resultados foram apresentados em gráficos para posterior discussão.

Os dados relativos à formação dos membros participantes do GESTE foram identificados por meio de buscas online de informação de domínio público, não sendo necessária a assinatura de termo de consentimento livre e esclarecido e a submissão do projeto ao Comitê de Ética.

\section{RESULTADOS}

Desde o início de sua formação, o grupo contou com 56 participantes. Desses, seis são homens e 50 são mulheres, totalizando $89 \%$ de participantes do sexo feminino. Com relação à formação na graduação, aproximadamente $95 \%$ dos participantes eram enfermeiros e $5 \%$ dos participantes eram graduados em terapia ocupacional, fisioterapia e biologia.

Com relação ao grau máximo de formação acadêmica dos participantes do grupo, temos o gráfico a seguir: 
Gráfico 1 - Grau máximo de formação acadêmica dos membros do grupo. São Paulo, 2015.

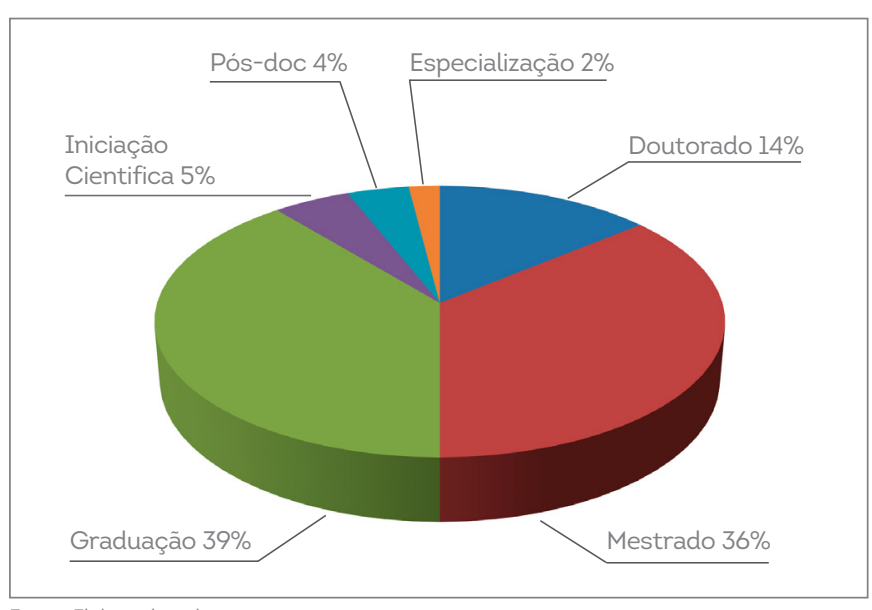

Fonte: Elaborado pelo autor

Verifica-se no gráfico acima que apenas dois membros do grupo (4\%) obtiveram formação no pós-doutorado. Ao descrevermos as porcentagens mais significativas, notamos que quase $40 \%$ dos integrantes desse GP concluiu a graduação, seguidos daqueles que obtiveram titulação de mestres, que contabilizaram $36 \%$ dos participantes. Ainda, concluíram o doutorado $14 \%$ dos membros, iniciação científica $5 \%$ e especialização $2 \%$.

Com relação à inserção no mercado de trabalho, após a titulação, observamos que dois membros com pós-doutorado estão inseridos, como docentes, em universidades públicas. Dos oito membros, com título de Doutor, que trabalham como docentes no ensino superior de Enfermagem, seis lecionam em universidades federais, um em universidade estadual e um em uma faculdade privada. Notamos uma tendência dos participantes com maior graduação em partirem para o ensino superior em instituições públicas, em que, para o ingresso, exige-se o título de Doutor.

Dos integrantes do grupo com título de Mestre, nove atuam na área acadêmica, inseridos na docência em faculdades e/ ou universidades privadas; sete integrantes trabalham na área assistencial. Desses, cinco prestam serviço em hospitais universitários ou vinculados a instituições de ensino: dois dos integrantes acumulam dois vínculos, sendo um assistencial e outro de professor em nível médio e superior. Um membro atua apenas como pesquisador e dois membros não têm mais contato com o grupo de pesquisa, não tendo sido encontrados seus dados.

Considerando a pós-graduação stricto sensu, o gráfico a seguir mostra a frequência de formação de mestres e doutores no decorrer do período, por meio das teses e dissertações defendidas.
Gráfico 2 - Formação stricto sensu dos membros do grupo. São Paulo, 2015.



Fonte: Elaborado pelo autor

O gráfico permite observar a irregularidade na formação de mestres e doutores. No período de 1997 a 1999, houve formação somente de mestres, sendo que o primeiro título de doutor surge no segundo triênio, entre 2000 e 2002. No período de 2003 a 2005, a formação de ambos se iguala e nos periodos seguintes a formação de doutores é incrementada, enquanto a formação de mestres diminui.

\section{DISCUSSÃO}

Uma das maneiras dos pesquisadores obterem uma formação consistente em pesquisa, em um dado campo de estudo, é a sua participação ativa em GP, considerando que neles ocorre o encontro fecundo do saber científico com os aspectos práticos vivenciados no cotidiano dos diversos pesquisadores ${ }^{(10)}$.

A respeito do GP em questão, percebemos que a predominância de participantes do gênero feminino se assemelha ao perfil da enfermagem no cenário nacional(11). Isso decorre do fato dos pesquisadores estarem inseridos em profissões tipicamente femininas como enfermagem e docência. Leva-se em consideração que o GP tem sua criação e operação na Escola de Enfermagem da Universidade de São Paulo (EE-USP), instituição que agrega as duas profissões. Além dessa questão, notamos interesse dos graduados em dar continuidade à formação acadêmica, visto o vínculo já estabelecido com os membros do grupo, conhecendo suas linhas de pesquisa.

O corpo docente nos grupos de pesquisa é considerado como a base de sustentação na construção e materialização de conhecimento em pesquisa. É graças a ele que a evolução do saber científico ocorre, na medida em que se atribui aos docentes a orientação, o compartilhamento e a difusão do 
conhecimento juntamente com seus membros. Tal aspecto evidencia a relevância do docente como sujeito que contribui para a evolução da ciência e tecnologia, cumprindo com sua responsabilidade social frente aos investimentos recebidos pelos órgãos de fomento em pesquisa ${ }^{(12)}$.

Referente ao grau máximo de formação dos participantes desse GP, notamos neste estudo que ainda se formam mais mestres do que doutores e a oferta de vagas para mestrado nos programas de pós-graduação em enfermagem também é maior do que as vagas para doutorado(13). Isso porque, muitas vezes, a formação dos mestres segue a formação de doutores. Mesmo assim, a formação dos doutores é crescente nos últimos biênios, uma vez que é crescente a formação de recursos humanos, ou seja, orientadores dos programas de pós-graduação que podem assumir essa orientação. Essa realidade mostra a importância para a maior titulação de doutores, considerada requisito para concurso e inserção em universidades públicas. Notamos que a maioria dos doutores são absorvidos nessas instituições, compondo, assim, 85\% do seu corpo docente $\mathrm{e}^{(1,14)}$.

A formação de mestres e doutores, ainda, é estabelecida nos programas de pós-graduação, em decorrência da sua avaliação e qualificação, segundo critérios estabelecidos pela Coordenação de Aperfeiçoamento de Pessoal de Nível Superior (CAPES).

Considera-se, ainda, que os cursos de doutoramento no país são relativamente recentes e os mestrados mais antigos, instituídos na década de 1970(1).

Inferimos que uma das razões para o maior número de mestres esteja também relacionado ao menor dispêndio de tempo para a conclusão do curso, tornando-se, assim, uma opção mais viável para o estudante. Além disso, na maior parte das instituições de ensino, constitui curso precedente ao ingresso no doutorado.

Com relação aos mestres, mais da metade deles já ingressou em atividades docentes em instituições privadas, que permitem sua contratação sem título de doutor. A maioria dos mestres que se encontra na área assistencial atua em hospitais universitários e/ou instituições de ensino, onde desenvolvem atividades de supervisão em estágios de graduação e preceptoria em programas de residência. Tal aspecto evidencia que a titulação de mestres promove o crescimento profissional no seu local trabalho e confirma a tendência de que a pós-graduação stricto sensu também potencializa a formação do trabalhador da área de assistência, contribuindo para tornar sua prática mais reflexiva, inovadora e de melhor qualidade quando agregada aos conhecimentos advindos da pesquisa e da formação na academia $^{(2,13)}$.

Além disso, o crescimento profissional, como consequência dessa titulação, pode ser entendido como valorização do nível de conhecimento do trabalhador, trazendo-lhe assim mais motivação em seu ambiente laboral. Vale lembrar que, se sentindo reconhecido e motivado, o trabalhador de enfermagem percebe a importância do seu esforço, mesmo em situações de desgaste ${ }^{(15)}$.

Com base no perfil e evolução na carreira dos integrantes do grupo, observamos a potencialidade do grupo em contribuir não só para o desenvolvimento acadêmico, mas também para a formação de líderes assistenciais, considerando que a produção de conhecimento lhes oferece subsidios para desenvolver atividades de ensino, agregadas à assistência, tornando-os referências em conhecimento técnico-científico para os demais membros da equipe de enfermagem ${ }^{(16)}$.

Também, há, no grupo de pesquisa, a intenção de contribuir com o processo de formação dos enfermeiros, por meio da inserção de alunos de graduação em iniciação científica, desenvolvendo seus TCC. Considera-se desejável a capacidade de proporcionar múltiplos olhares para os diversos cenários a partir de um GP composto também por alunos de graduação ${ }^{(17)}$. O GP proporciona o fortalecimento do elo pesquisa-ensino, quando a inserção do aluno ocorre desde o período de graduação(8)

Em um GP, deve ser valorizada a presença de pesquisadores de diversos niveis de formação, considerando que essa heterogeneidade de saberes proporciona uma aprendizagem mais abrangente. Dessa forma, o GP começa a adquirir uma identidade própria que desenvolve um pensamento crítico coletivo, gerando maior segurança e autonomia de pesquisa(10)

Essa autonomia que se inicia em pesquisa é lapidada pela dinâmica do grupo, em que são discutidas as questões metodológicas, os referenciais teóricos, bem como o tratamento dos dados coletados, que são partilhados entre alunos e pós-graduandos, atrelados à experiência trazida pelos docentes na liderança do grupo. A emancipação dos membros, futuros líderes de GPs, pode ser avaliada pelos docentes, considerando os focos de aprendizado em pesquisa, evidenciada pelo interesse, conhecimento do referencial e método, criatividade, trabalho em equipe e identidade adquirida(18).

\section{CONCLUSÃO}

Os GPs e a formação nesse locus fortalece o papel do enfermeiro nas áreas acadêmica e assistencial. $O$ avanço de conhecimento proporcionado pelo incremento na produção científica permite aos enfermeiros trilhar caminhos de maior qualificação e valoração em suas práticas, agregando maior visibilidade à profissão e possibilitando a disseminação do conhecimento adquirido em pesquisa. 
Naárea de saúde do trabalhador, a progressão na formação se reflete na criação e implementação de alternativas inovadoras para melhorias nas condições de trabalho e fora dele e, também, na formação de outros trabalhadores.

Os achados deste estudo corroboram com a literatura, em que predominam nos GPs mulheres que, com as maiores titulações, optaram por dedicar-se ao ensino, seja em sala de aula ou em campo. Ainda, são extremamente valorizadas as participações de alunos de graduação, de modo a manter a continuidade no processo de ensino-aprendizagem em pesquisa e assegurar vanguardismo, qualidade e avanço nos elos entre assistência e pesquisa.

Em relação à absorção dos pesquisadores pelo mercado de trabalho, observamos o maior número de doutores nas instituições públicas de ensino, enquanto que os mestres atuam, em sua maioria, em faculdades particulares ou em instituições hospitalares também vinculadas ao ensino e pesquisa.

\section{REFERÊNCIAS}

1. Scochi CGS, Munari DB, Gelbcke FL, Erdmann AL, Gutiérrez MGR, Rodrigues RAP. Pós-graduação Stricto Sensu em Enfermagem no Brasil: avanços e perspectivas. Rev Bras de Enferm [Internet]. 2013 [cited 2017 Abr 23]:6:680-89. Available from: http://www.scielo.br/scielo.php?script=sci_arttextEpid=S0034-71672013000700011

2. Fortuna CM, Mishima SM. A pesquisa de enfermagem e a qualificação da assistência: algumas reflexões. Rev Eletr Enf [Internet]. 2012 [cited 2017 Abr 25];14(4):740-2. Available from: https://www.revistas.ufg.br/ fen/article/view/13408

3. Godinho MR, Greco RM, Gonçalves AMC. Enfermagem e saúde do trabalhador: análise dos grupos de pesquisa. Rev Rene [Internet]. 2011 [cited 2017 Abr 25];12(4):825-32. Available from: http://www.revistarene.ufc.br/vol12n4_html_site/a21v12n4.html

4. Erdmann AL, Peiter CC, Lanzoni, GMM. Grupos de pesquisa em enfermagem no Brasil: comparação dos perfis de 2006 e 2016. Rev Gaúcha Enferm [Internet]. 2017 [cited 2017 Mai 02];38(2):e69051. Available from: http://dx.doi.org/10.1590/1983-1447.2017.02.69051

5. Silva SM, Telles ACM, Gallasch CH, Almeida MCS, Baptista PCP, Fell VEA. Temáticas investigadas pelo Grupo de Estudos sobre a Saúde do Trabalhador de Enfermagem e Saúde. Rev enferm UERJ [Internet] 2016 [cited 2017 Mai 02];24(5):e22974. Available from: http://www.e-publicacoes.uerj.br/index.php/enfermagemuerj/article/view/22974

6. Baptista PCP. Pustiglione M. Almeida MCS, Felli VEA, Garzin ACA Melleiro MM. Saúde dos trabalhadores de enfermagem e a segurança do paciente: o olhar de gerentes de enfermagem. Rev Esc Enferm USP [Internet]. 2015 [cited 2017 Mai 05]; 49(Esp2):122-8. Available from: http://www.scielo.br/pdf/reeusp/v49nspe2/1980-220X-reeusp-49-spe2-0122.pdf

7. Soares MI, Resck ZMR, Camelo SHH, Lima GS. A pesquisa como fio condutor para a produção do cuidado em enfermagem: revisão integrativa da literatura. Revista Eletrônica Gestão \& Saúde [Internet] 2015 [cited 2017 Mai 05];6(1):591-605. Available from: http://periodicos. unb.br/index.php/rgs/article/view/13755/9689

8. Azevedo DM, Holanda CSM, Costa RKS. A importância do grupo de pesquisa na formação em enfermagem: uma experiência na graduação. Saúde transform soc [Internet]. 2013 [cited 2017 Mai 15]:4(1):1-2. Available from: http://incubadora.periodicos.ufsc.br/index.php/saudeetransformacao/article/view/1992/2488

9. Araújo AML, Morais HCC, Vasconcelos HCA, Rabelo JC, Santos RXL, Holanda RE. A pesquisa cientifica na graduação em enfermagem e sua importância na formação profissional. Rev enferm UFPE [Internet] 2015 [cited 2017 Mai 15];9(9):9180-7. Available from: https://periodicos. ufpe.br/revistas/revistaenfermagem/article/viewFile/10716/11800
10. Justina LAD, Caldeira AMA. A pesquisa de iniciação científica como espaço de formação inicial de professores e pesquisadores de Biologia. Revista Electrónica de Investigación en Educación en Ciencias [Internet]. 2012 [cited 2017 Mai 18];7(2);1-12. Available from: http://www.scielo.org.ar/img/revistas/reiec/v7n2/html/v7n2aOl.htm

11. Machado MH, Filho WA, Lacerda WF, Oliveira E, Lemos W. Wemelinger $M$, et al. Caracteristicas gerais da enfermagem: o perfil sociodemgráfico. Enferm. Foco [Internet]. 2016 [cited 2107 Mai 20];7(spe):09-14. Available from: http://revista.portalcofen.gov.br/index.php/enfermagem/article/view/686/296

12. Oliveira AC, Ferreira MA. O papel estratégico do corpo docente nos programas de pós-graduação. Esc Anna Nery (imp.) [Internet]. 2011 [cited 2017 Mai 25];15(2):227-9. Available from: http://www.scielo.br/pdf/ ean/v15n2/en_v15n2aOl.pdf

13. Erdmann AL. Formação de especialistas, mestres e doutores em enfermagem: avanços e perspectivas. Acta Paul Enferm [Internet]. 2009 [cited 2017 Mai 25];22(spel):551-3. Available from: http://www.scielo.br/ pdf/ape/v22nspel/21.pdf

14. Cunha MI, Zanchet BBA, Ribeiro GM. Qualidade do ensino de graduação: culturas, valores e seleção de professores. Práxis Educativa [Internet]. 2013 [cited 2017 Mai 31]:8(1):219-41. Available from: http://177.101.17.124/index.php/praxiseducativa/article/ view/5377/3476

15. Corral-Mulato S, Bueno, SMV. Carreira docente de enfermagem: escolhas e significados arq. Cienc. Saúde UNIPAR [Internet]. 2014 [cited 2017 Jun 05]:18( 3):169-172. Available from: http://www.revistas. unipar.br/index.php/saude/article/view/5192/3004

16. Andrade D, Alves MB, Gobo DC, Silva EA, Baia WRM. Implantação do Modelo Enfermeiro Referência "Primary Nursing" em um Hospital Público Oncológico no Estado de São Paulo. Blucher Medical Proceedings [Internet]. 2014 [cited 2017 Jun 10]:1(2):64. Available from: http:// www.proceedings.blucher.com.br/article-details/implantao-do-modelo-enfermeiro-referncia-primary-nursing-em-um-hospital-pblico-oncolgico-no-estado-de-so-paulo-9485

17. Erdmann AL, Mello, Ferreira AL, Andrade SR, Klock P. Funcionalidade dos Grupos de Pesquisa de Administração/Gestão/ Gerência de Enfermagem. Rev Rene [Internet]. 2010 [cited 2017 Jun 20]:11(2):1926. Available from: http://www.revistarene.ufc.br/vollln2_html_site/ aO2vlln2.htm

18. Teixeira LA, Passos MM, Arruda SM. A formação de pesquisadores em um grupo de pesquisa em Educação em Ciências e Matemática. Ciênc. Educ [Internet]. 2015 [cited 2017 Jun 25];21(2):525-41. Available from: http://www.redalyc.org/articulo.oa?id=251038426016 